\title{
Screen-printed EMI shielding materials on Mulberry paper for wearable electronics with high mechanical strength
}

\author{
Hoseong, Sooman (Hoseong Song, Sooman Lim) \\ Graduate School of Flexible Printing Electronics, Chonbuk National University
}

\begin{abstract}
The wearable and flexible printed electronics has shown remarkable advancement due to its low fabrication cost and high performance of devices. The screen printing process for wearable electromagnetic interference shielding (EMI) electronics were profitable among the other printing methods. Herein, we fabricated the silver flakes based EMI shielding device on the mulberry paper using facile screen printing method and investigated their optimization condition with electrical conductivity and EMI shielding effectiveness. The $2 \mathrm{~mm}$ squeeze depth shows the lowest sheet resistance of $60 \mathrm{~m} \Omega$ and the excellently coated silver flaks on mulberry paper were confirmed by SEM. In addition, the mechanical properties of the fabricated EMI shielding device was improved due to longer fiber length than other commercial papers. The optimized screen printing process shows best printing method for wearable EMI shielding.
\end{abstract}

Keywords: Silver flakes, Mulberry paper, Screen printing, electrical and mechanical properties, EMI shielding. 